\title{
Analysis of the expression and potential molecular mechanism of interleukin-1 receptor antagonist (IL1RN) in papillary thyroid cancer via bioinformatics methods
}

Zhenyu Xie ${ }^{\dagger}$, Xin Li ${ }^{\dagger}$, Yuzhen He, Song Wu, Shiyue Wang, Jianjian Sun, Yuchen He, Yu Lun, Shijie Xin and Jian Zhang*

\begin{abstract}
Background: Interleukin-1 receptor antagonist (IL1RN) has been reported as a biomarker of many cancers. However, the biological function of IL1RN in papillary thyroid carcinoma (PTC) remains undetermined.

Methods: We obtained IL1RN expression data from The Cancer Genome Atlas (TCGA) database. Enrichment analysis of coexpressed genes and ILIRN methylation analysis were performed via LinkedOmics. The correlations between ILIRN and immune infiltrates were investigated via ESTIMATE, TIMER and TISIDB. We analyzed the association of IL1RN expression with pancancer overall survival (OS) via Gene Expression Profiling Interactive Analysis (GEPIA).

Results: IL1RN showed higher expression levels and lower methylation levels in PTC tissues than in normal tissues. Higher IL1RN expression was significantly associated with shorter progression-free survival (PFS), advanced tumor stage, tumor metastasis, increased incidence of BRAF mutations, and decreased incidence of N-RAS and H-RAS mutations. Genes coexpressed with ILIRN participate primarily in immune-related pathways. IL1RN expression positively correlated with immune infiltration, tumor progression and poor OS for all cancers.

Conclusions: IL1RN is a good prognostic and diagnostic biomarker for PTC. IL1RN may promote thyroid cancer progression through immune-related pathways. Methylation may act as an upstream regulator of IL1RN expression and biological function. Additionally, IL1RN was shown to have broad prognostic value in a pancancer cohort.
\end{abstract}

Keywords: IL1RN, Papillary thyroid carcinoma, Diagnostic biomarker, Prognostic biomarker, Cancer immunity, IL-1

\footnotetext{
* Correspondence: jianzhang@cmu.edu.cn

${ }^{\dagger}$ Zhenyu Xie and Xin Li contributed equally to this work.

Department of Vascular and Thyroid Surgery, The First Hospital, China

Medical University, Shenyang, China
}

C The Author(s). 2020 Open Access This article is licensed under a Creative Commons Attribution 4.0 International License, which permits use, sharing, adaptation, distribution and reproduction in any medium or format, as long as you give appropriate credit to the original author(s) and the source, provide a link to the Creative Commons licence, and indicate if changes were made. The images or other third party material in this article are included in the article's Creative Commons licence, unless indicated otherwise in a credit line to the material. If material is not included in the article's Creative Commons licence and your intended use is not permitted by statutory regulation or exceeds the permitted use, you will need to obtain permission directly from the copyright holder. To view a copy of this licence, visit http://creativecommons.org/licenses/by/4.0/. The Creative Commons Public Domain Dedication waiver (http://creativecommons.org/publicdomain/zero/1.0/) applies to the data made available in this article, unless otherwise stated in a credit line to the data. 


\section{Background}

Thyroid carcinoma (THCA) is the most common endocrine malignancy worldwide [1]. The incidence of THCA has increased sharply over the past 3 decades [2]. Papillary thyroid carcinoma (PTC) is the major subtype of THCA, accounting for more than $90 \%$ of cases [3]. The clinical course of PTC is generally indolent, and the cancerspecific mortality of PTC is low compared to that of other cancers [4]. However, the incidence of cervical lymph node metastasis is high, which leads to local recurrence and poor prognosis [5]. The main task for the future is to identify high-risk patients and to give them appropriate treatment and care [6]. Therefore, further studies are required to explore the underlying mechanisms of tumorigenesis as well as identify additional biomarkers that predict prognosis and serve as therapeutic targets.

The interleukin-1 (IL-1) family of cytokines are the most effective molecules in the innate immune system [7]. IL-1 receptor antagonist (IL1RN) was initially found as a natural antagonist of IL-1 [8]. IL1RN is structurally related to IL-l $\alpha$ and IL-1 $\beta$ but binds to IL- 1 receptors on various target cells without inducing any discernible biological responses [9]. The balance between IL1RN and IL-1 plays a crucial role in many diseases, including cancer [10]. IL1RN has been studied in several cancers, including prostate carcinoma [11, 12], cervical carcinoma [13], gastric carcinoma [14], bronchogenic carcinoma [15], endometrial cancer [16], lung cancer [17], ovarian cancer [18], oral malignancies [19], leukemia [20], and other cancers.

Two structural variants of IL1RN have been described: the soluble extracellular form (sIL-1ra) and the intracellular form (icIL-1ra) [8]. Niedzwiecki, S. and colleagues assayed the serum levels of IL1RN in thyroid cancer patients. They measured preoperative IL1RN serum levels of patients with thyroid cancer, and the results showed that the serum concentrations of sIL-1ra were significantly higher in anaplastic carcinoma (ATC) and follicular carcinoma (FTC) patients [21]. To our knowledge, no reports have been published to date concerning IL1RN expression in thyroid tissue. Because IL1RN has been associated with various diseases and the serum concentration of sIL-1ra has been confirmed to be increased in PTC, we hypothesized that IL1RN may play a role in PTC. The objective of this study was to investigate IL1RN expression in normal and PTC tissues by performing bioinformatics analysis to elucidate its possible role in tumor progression.

\section{Methods}

\section{Expression level analysis}

The cohort that comprised 512 PTC and 58 normal thyroid samples was obtained from The Cancer Genome
Atlas (TCGA) (https://tcga-data.nci.nih.gov/tcga/). The clinical data, normalized RNA expression data, DNA methylation data and simple nucleotide variation data were downloaded from the TCGA data portal.

To assess the diagnostic value of IL1RN, we selected data-sets containing both PTC and normal tissues in the GEO database. The gene expression profiles of four independent datasets (GSE3467, GSE33630, GSE58545, and GSE60542) were downloaded from the National Center for Biotechnology Information (NCBI) Gene Expression Omnibus (GEO) database (http://www.ncbi. nlm.nih.gov/geo/).

\section{Diagnostic and prognostic value analysis}

ROC curves were plotted, and the area under the ROC curve was calculated using the ROCR package in $\mathrm{R}$. The patients were divided into a high IL1RN expression group (H-IL1RN) and a low IL1RN expression group (L-IL1RN) according to the best matched value for the survival analysis. The best cut-off value was derived using Cutoff Finder software based on an $R$ routine which optimized the significance of the split between Kaplan-Meier (K-M) survival curves measured by the log-rank test [22]. K-M survival curves were generated by the $\mathrm{R}$ survival package. The primary end point of the study was progression-free survival (PFS). Univariate and multivariate analyses were performed using the Cox regression model to assess the significance of various variables to survival. A chi-square test was performed to compare the clinical characteristics between the H-IL1RN group and the L-IL1RN group.

\section{Gene functional enrichment analysis}

We identified the genes that were significantly positively or negatively correlated with IL1RN using the LinkedOmics website (http://www.linkedomics.org/) [23].

The top 50 positively correlated genes and the top 50 negatively correlated genes were selected to build the heatmaps.

These genes were input into the GO and KEGG websites to obtain the enriched GO terms and significant KEGG pathways. GO function annotation analysis was performed based on the GO database (http://geneontology.org/page/go-database), and KEGG pathway annotation analysis was performed based on the KEGG database (http://www.kegg.jp/kegg/ko.html).

The protein-protein interaction (PPI) network with a confidence > 0.7 was constructed using STRING (https:// string-db.org) and CytoScape version 3.7.2.

Tumor immunology analysis

ESTIMATE used the single-sample gene-set enrichment analysis (ssGSEA) score to quantify the 
enrichment levels of immune gene signatures in tumors. ESTIMATE [24], a method that uses gene expression signatures to infer the fractions of stromal and immune cells in tumor samples, was used to evaluate the levels of immune cell infiltration (immune score), the stromal content (stromal score), the stromal-immune comprehensive score (ESTIMATE score) and tumor purity for each THCA sample.

The Tumor Immune Estimation Resource (TIMER) web server (https://cistrome.shinyapps.io/timer/), a comprehensive analytic web tool [25], was used to analyze the correlation of IL1RN with infiltration of immune cells, including B cells, CD8+ T cells, CD4+ $\mathrm{T}$ cells, macrophages, neutrophils and dendritic cells (DCs).

We used TISIDB (http://cis.hku.hk/TISIDB/), a web portal for investigation of tumor and immune system interaction [26], to determine the Spearman correlation between IL1RN expression and 28 TIL types, chemokines, immune-activating cytokines, immunosuppressive cytokines, major histocompatibility complex (MHC) molecules, and chemokine receptors.

\section{Methylation-related analysis}

DNA methylation data were downloaded from the data portal of TCGA (https://portal.gdc.cancer.gov/), and the DNA methylation levels of the PTC and control groups were then compared. Spearman correlation analysis was conducted to examine the associations between the methylation density and gene expression and between the methylation density and tumor purity. Additionally, we analyzed the correlation between IL1RN expression and the methylation level of each CpG site using the Spearman correlation test. The analysis of the relationship between methylation and the clinical characteristics and the GO and KEGG analyses were performed using linkedOmics.

\section{Pancancer analysis}

The pancancer expression and survival analysis of IL1RN was performed using the online software Gene Expression Profiling Interactive Analysis (GEPIA) (http://gepia. cancer-pku.cn/) [27]. We used TISIDB to analyze the relationships between IL1RN expression and overall survival (OS) in pancancer. K-M curves were generated for pancancer using the TCGA cohort data.

\section{Related mutant gene analysis}

The analysis of the relationship between IL1RN expression and PTC mutations was performed by linkedOmics. The IL1RN expression level was further compared between the groups with wild-type and mutant versions of BRAF, NRAS and HRAS.

\section{Statistical analysis}

The $t$-test and Mann-Whitney $U$ test were used for comparisons between two groups. The chi-square test was used to assess the differences in clinical parameters between the L-IL1RN and H-IL1RN groups. The Pearson and Spearman methods were used for correlation analysis. The log-rank method was used to calculate the significant $P$-values related to survival. $\mathrm{R}$ software (v3.6.0) and SPSS version 25.0 software were used for statistical processing. Visualization of data was performed with GraphPad Prism V.8.0 and R software. $P<0.05$ was considered significant.

\section{Results}

The differential expression and diagnostic value of IL1RN The expression levels of IL1RN were initially analyzed in the TCGA cohort consisting of 512 PTC samples and 58 normal samples (Fig. 1a). Furthermore, expression data from four independent PTC cohorts obtained from the Gene Expression Omnibus (GEO) (GSE3467, GSE33630, GSE58545, and GSE60542) were employed for validation (Fig. 1d-g). Both the GEO and TCGA patient cohorts had significantly higher IL1RN mRNA expression in PTC tissues than in normal tissues $(P<0.05)$.

ROC curves were constructed to evaluate the diagnostic value of IL1RN for PTC. The area under the ROC curve of the TCGA cohort was 0.7084 (Fig. 1b) and that of each GEO cohort was 0.9753, 0.7279, 0.8868 , and 0.8384 , respectively (Fig. $1 \mathrm{~h}-\mathrm{k}$ ). These results suggest the good positive diagnostic value of IL1RN for PTC.

\section{Prognostic value and clinical significance of IL1RN}

The PTC samples from the TCGA cohort were divided into two groups according to the IL1RN expression level: the high-expression group (H-IL1RN) $(n=116)$ and the low-expression group (L-IL1RN) $(n=385)$. Differences in progression-free survival (PFS) between the two groups were analyzed by Kaplan-Meier analysis (Fig. 1c). The results showed that patients with high IL1RN expression had significantly shorter PFS than those with low expression $(P=0.0034)$. However, the data for the multivariate analysis by the Cox regression model suggested that IL1RN expression was not a significant independent prognostic risk indicator for PTC (hazard ratio, 1.033; 95\% confidence interval, 0.999$1.068, P>0.05$ ) (Table 1). Age was an independent predictor of poor prognosis $(P<0.05)$.

To examine the clinical significance of IL1RN in PTC, the relationship between the expression level of IL1RN and clinical characteristics was investigated by the chisquare test (Table 2). The results suggested that IL1RN expression was significantly associated with clinical 
A

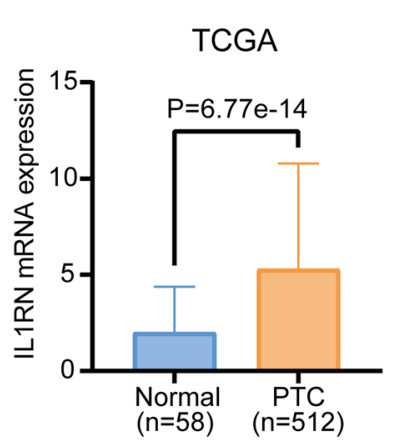

C

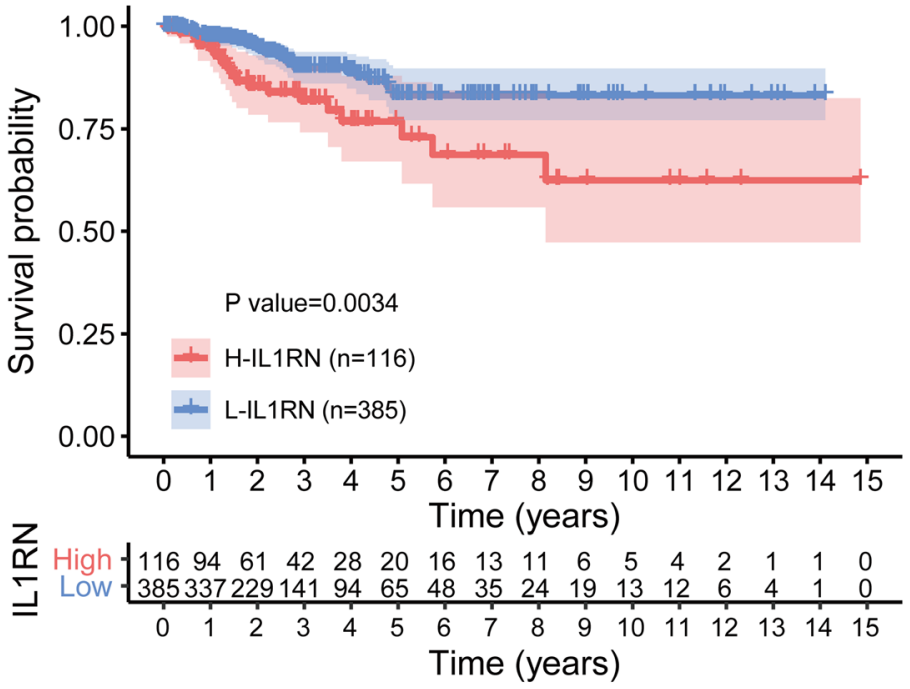

B

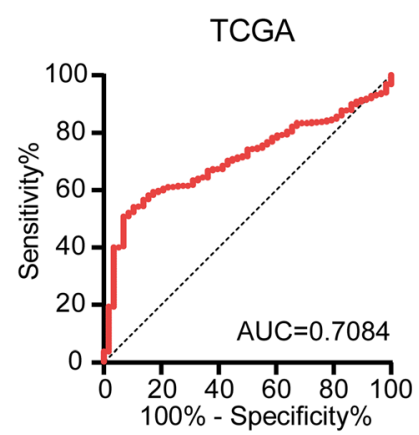

Time (years)
D

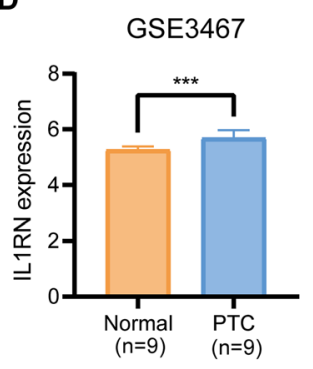

H<smiles>CCC[Se]C#[Ge]</smiles>

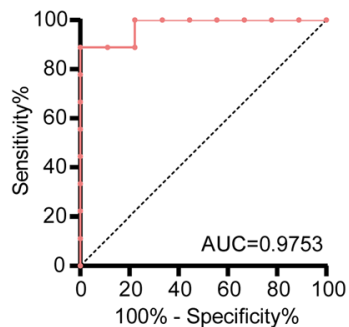

E

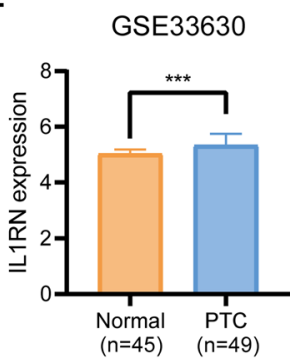

I

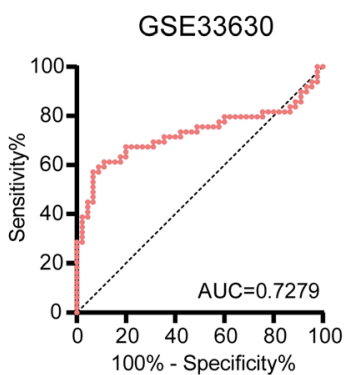


(See figure on previous page.)

Fig. 1 Diagnostic value of ILIRN in PTC. a Comparison of IL1RN mRNA expression between PTC and normal tissues in TCGA. b Diagnostic efficacy of IL1RN as shown by the ROC curve. c Kaplan-Meier curve for progression-free survival (PFS) in H-IL1RN group and L-IL1RN group. $\mathbf{d}-\mathbf{g}$ mRNA expression of IL1RN in 4 GEO verification cohorts (GSE3467, GSE33630, GSE58545, GSE60542). (H-K) ROC curve showing the diagnostic performance of IL1RN expression in the verification cohorts. ${ }^{*} P<0.05$, ${ }^{* *} P<0.01$, ${ }^{* *} P<0.001$, ${ }^{* * * *} P<0.0001$ here and in the following figures

stage, $\mathrm{N}$ stage, $\mathrm{T}$ stage, pathologic type, BRAF mutations and RAS mutations.

\section{Gene coexpression and pathway enrichment analysis}

The Function module of LinkedOmics was used to analyse mRNA sequencing data from 512 PTC patients in TCGA. The result is presented as a volcano plot (Fig. 2a). A total of 8960 genes showed significant positive correlations with IL1RN(red dots), while 10,967 genes showed significant negative correlations with IL1RN (green dots). The top 50 positively (Fig. 2b) or negatively (Fig. 2c) correlated genes are depicted by heatmaps. In addition, 2 hub genes (CCL20 and FN1) were identified from the protein-protein interaction (PPI) network (Fig. 2d).

We used gene set enrichment analysis (GSEA) to conduct GO term and KEGG analyses for all positive IL1RN co-expressed or negative IL1RN co-expressed genes. GO enrichment analysis revealed that IL1RN co-expressed genes were mainly enriched for the GO biological process terms "neutrophil mediated immunity", "positive regulation of cytokine production", "adaptive immune response" and "immune responseregulating signalling pathway" (Fig. 2e). Further KEGG enrichment analysis suggested that the identified genes were mainly involved in pathways associated with "cell adhesion molecules (CAMs)", "cytokine-cytokine receptor interaction", and "phagosome" (Fig. 2f).

\section{Immune-related analysis of IL1RN}

Using ESTIMATE, the association between IL1RN expression and immune infiltrates was analyzed. We found that the H-IL1RN group showed a higher immune score (Fig. 3b) and ESTIMATE score (Fig. 3c) and lower tumor purity (Fig. 3d) than the L-IL1RN group. No significant difference in the stromal score was found between the two groups (Fig. 3a). The correlations between the IL1RN expression level and the infiltration of six immune cell types in PTC were estimated. The results demonstrated that IL1RN expression was found to be significantly positively correlated with the infiltration of immune cells, especially neutrophils and dendritic cells (Fig. 3e). Correlations between IL1RN expression and 28 TIL types (Fig. 3f), chemokines (Fig. 3g), immune-activating cytokines (Fig. S1A), immunosuppressive cytokines (Fig. S1B), MHC molecules (Fig. S1C), and chemokine receptors (Fig. S1D) in pancancer are shown in heatmaps. A positive correlation between IL1RN expression and immune-related molecules was observed.

\section{Methylation-related analysis}

The data showed that the degree of IL1RN DNA methylation was lower in carcinoma tissues than in normal tissues $(P<0.0001)$ (Fig. 4a). To further determine the role of methylation, we performed a correlation analysis between the DNA methylation levels of IL1RN and the expression levels of IL1RN. As expected, DNA methylation negatively correlated with IL1RN expression $(\mathrm{R}=-0.600, P=2.29 \mathrm{e}-50)$ (Fig. $4 \mathrm{~b})$. Tumor purity was significantly positively associated with IL1RN methylation ( $\mathrm{R}=0.447, P=7.49 \mathrm{e}-32)$ (Fig. 4c). Patients with an advanced clinical stage (Fig. 4d), advanced $\mathrm{T}$ stage (Fig. 4e) and lymph node metastasis (Fig. 4f) tended to have decreased IL1RN methylation levels. In the pathological subtype of PTC, the tall cell type variant of PTC had the lowest degree of methylation, followed by the classical type, and the follicular variant of PTC had the highest degree of methylation (Fig. 4g).

Table 1 Results of univariate and multivariate logistic regression analysis

\begin{tabular}{|c|c|c|c|c|}
\hline \multirow[t]{2}{*}{ Variables } & \multicolumn{2}{|l|}{ Univariate analysis } & \multicolumn{2}{|l|}{ Multivariate analysis } \\
\hline & Hazard ratio $(95 \% \mathrm{Cl})$ & $P$ value & Hazard ratio $(95 \% \mathrm{Cl})$ & $P$ value \\
\hline Age $(y \geq 55 /<55)$ & $3.958(2.274-6.891)$ & $<0.001$ & $2.582(1.312-5.083)$ & 0.006 \\
\hline Sex & $1.471(0.834-2.592)$ & 0.182 & $1.098(0.604-1.997)$ & 0.758 \\
\hline Clinical stage & $1.736(1.380-2.185)$ & $<0.001$ & $1.301(0.968-1.748)$ & 0.081 \\
\hline Radiation therapy & $1.305(0.728-2.343)$ & 0.372 & $1.205(0.651-2.233)$ & 0.553 \\
\hline IL1RN & $1.058(1.024-1.093)$ & $<0.001$ & $1.033(0.999-1.068)$ & 0.059 \\
\hline
\end{tabular}


Table 2 Association between IL1RN expression and clinical parameters

\begin{tabular}{|c|c|c|c|}
\hline Clinical parameters & $\begin{array}{l}\text { L-IL1RN } \\
(n=385, \%)\end{array}$ & $\begin{array}{l}\text { H-IL1RN } \\
(n=116, \%)\end{array}$ & P-value \\
\hline \multicolumn{4}{|l|}{ Age $(y)$} \\
\hline$<55$ & $261(67.8)$ & $73(62.9)$ & \multirow[t]{2}{*}{0.330} \\
\hline$\geq 55$ & $124(32.2)$ & $43(37.1)$ & \\
\hline \multicolumn{4}{|l|}{ Sex } \\
\hline Female & $282(73.2)$ & $84(72.4)$ & \multirow[t]{2}{*}{0.859} \\
\hline Male & $103(26.8)$ & $32(27.6)$ & \\
\hline \multicolumn{4}{|l|}{ Clinical stage } \\
\hline । & $224(58.5)$ & $57(49.1)$ & \multirow[t]{5}{*}{0.001} \\
\hline$\|$ & $47(12.3)$ & $5(4.3)$ & \\
\hline III & $77(20.1)$ & $34(29.3)$ & \\
\hline IV & $35(9.1)$ & $20(17.2)$ & \\
\hline NA & 2 & 0 & \\
\hline \multicolumn{4}{|l|}{ Metastasis } \\
\hline MO & $208(97.2)$ & $74(96.1)$ & \multirow[t]{3}{*}{0.635} \\
\hline M1 & $6(2.8)$ & $3(3.9)$ & \\
\hline NA & 171 & 39 & \\
\hline \multicolumn{4}{|l|}{$\mathrm{N}$ classification } \\
\hline NO & $189(54.8)$ & $40(37.7)$ & \multirow[t]{3}{*}{0.002} \\
\hline N1 & $156(45.2)$ & $66(62.3)$ & \\
\hline NA & 40 & 10 & \\
\hline \multicolumn{4}{|l|}{ T classification } \\
\hline $\mathrm{T} 1$ & $120(31.2)$ & $22(19.3)$ & \multirow[t]{5}{*}{$<0.001$} \\
\hline $\mathrm{T} 2$ & $135(35.1)$ & $29(25.4)$ & \\
\hline T3 & $116(30.1)$ & $54(47.4)$ & \\
\hline T4 & $14(3.6)$ & $9(7.9)$ & \\
\hline NA & 0 & 2 & \\
\hline \multicolumn{4}{|l|}{ Pathologic type } \\
\hline Classical & $268(69.6)$ & $87(75.0)$ & \multirow[t]{4}{*}{$<0.001$} \\
\hline Follicular & $94(24.4)$ & $7(6.0)$ & \\
\hline Tall Cell & $17(4.4)$ & 19 (16.4) & \\
\hline Other & $6(1.6)$ & $3(2.6)$ & \\
\hline \multicolumn{4}{|l|}{ BRAF } \\
\hline Wild & $179(48.5)$ & $16(14.3)$ & \multirow[t]{3}{*}{$<0.001$} \\
\hline Mutation & $190(51.5)$ & $96(85.7)$ & \\
\hline NA & 16 & 4 & \\
\hline \multicolumn{4}{|l|}{ RAS } \\
\hline Wild & 311 (84.3) & $110(98.2)$ & \multirow[t]{3}{*}{$<0.001$} \\
\hline Mutation & $58(15.7)$ & $2(1.8)$ & \\
\hline NA & 16 & 4 & \\
\hline
\end{tabular}

To understand the biological significance of IL1RN methylation in THCA, a functional module of LinkedOmics was used to examine IL1RN coexpression pattern in the THCA cohort. Based on RNAseq, we screened 19, 927 genes related to IL1RN methylation (false discovery rate $($ FDR $<0.01)$. The GO (biological process) analysis results derived by GSEA were significant. The results indicated that IL1RN methylation coexpressed genes that participate primarily in mitochondrial gene expression, the generation of precursor metabolites and energy and small molecule catabolic process, while immune related activities, such as the adaptive immune response, immune response-regulating signalling, and neutrophil mediated immunity were inhibited (Fig. 4h). KEGG pathway analysis also showed that genes related to valine, leucine and isoleucine degradation, carbon metabolism and thermogenesis, among other pathways, were inhibited (Fig. 4i). These results illustrate that methylated IL1RN may inhibit immune-related pathways by downregulating IL1RN expression. Furthermore, the most significant methylation sites are shown in Table 3.

\section{Related mutant gene analysis}

The analysis of the relationship between IL1RN expression and PTC mutations was performed by LinkedOmics (Fig. S2A). The IL1RN expression level was further compared between the groups with wild-type and mutant variants of BRAF, NRAS and HRAS (Fig. S2B-D). The expression of IL1RN was significantly higher in PTC with the BRAF mutant than in PTC with the wild type (Fig. S2B). However, mutated NRAS (Fig. S2C) and mutated HRAS (Fig. S2D) correlated with decreased IL1RN expression.

\section{Pancancer analysis of IL1RN}

To investigate whether IL1RN has broad value, we performed a series of studies on IL1RN across all cancers. We analyzed the IL1RN expression levels in different kinds of tumors via the GEPIA platform (Fig. 5a). The results showed that the expression levels of IL1RN varied greatly in different cancer types. Of the 33 cancer types tested, 12 cancer types were associated with significantly increased IL1RN expression. Subsequently, the relationship between IL1RN and OS in pancancer was investigated (Fig. 5b). Kaplan-Meier survival curves for the high IL1RN expression group (4745 patients) and low IL1RN expression group (4750 patients) indicated that increased IL1RN expression was associated with a shorter survival time in pancancer $(\mathrm{HR}=1.6, P<$ 0.0001) (Fig. 5c). The results showed that increased IL1RN expression levels were significantly associated with shorter OS in kidney renal clear cell carcinoma (KIRC) (Fig. 5d), brain lower grade glioma (LGG) 


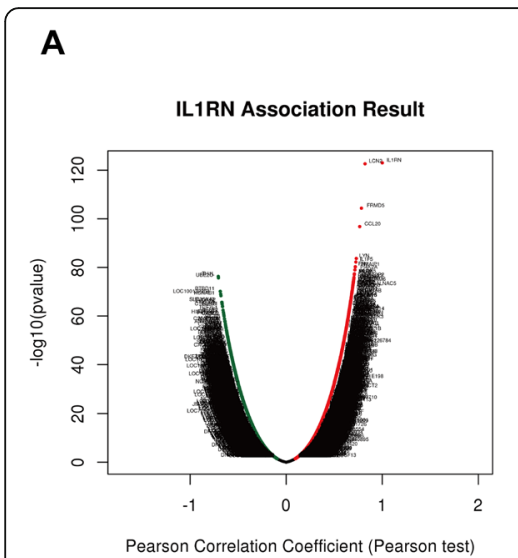

B

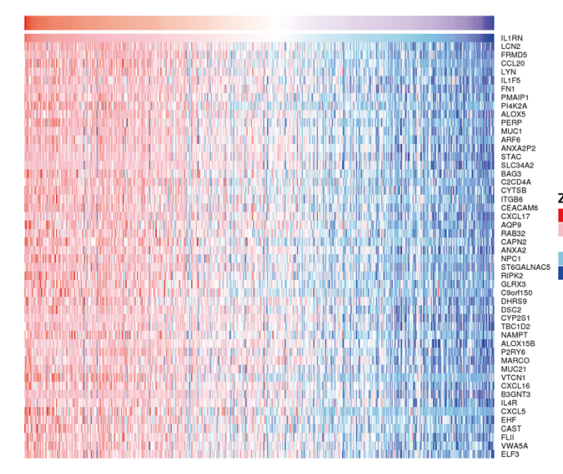

\section{C}

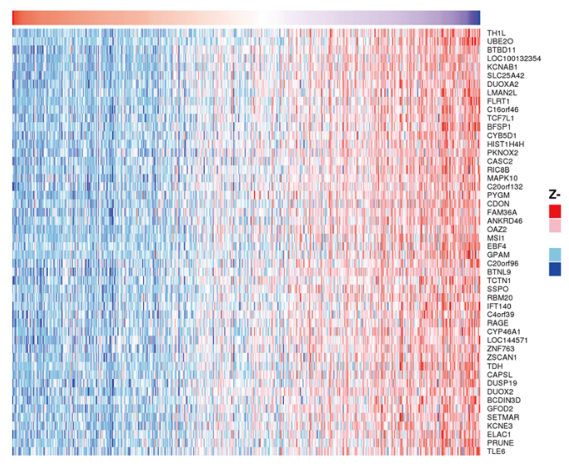

D

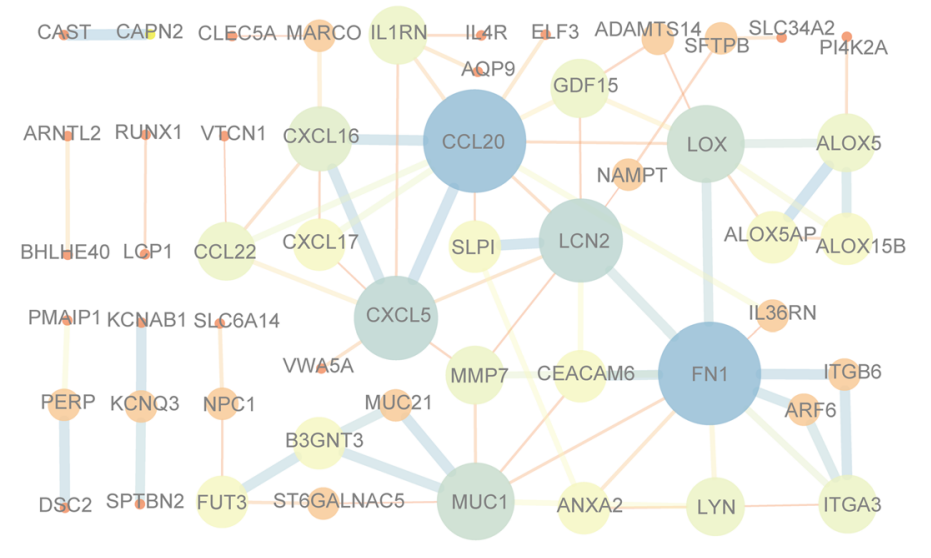

E

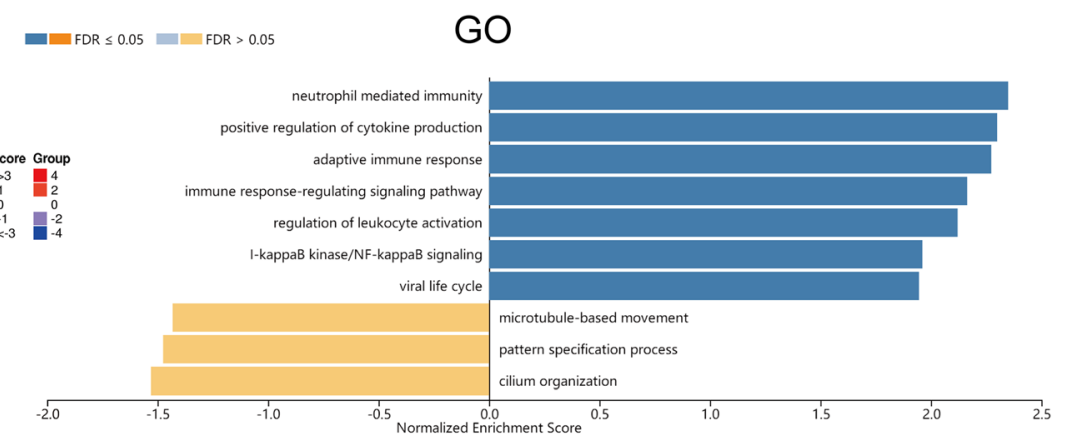

$\mathbf{F}$

\section{KEGG}

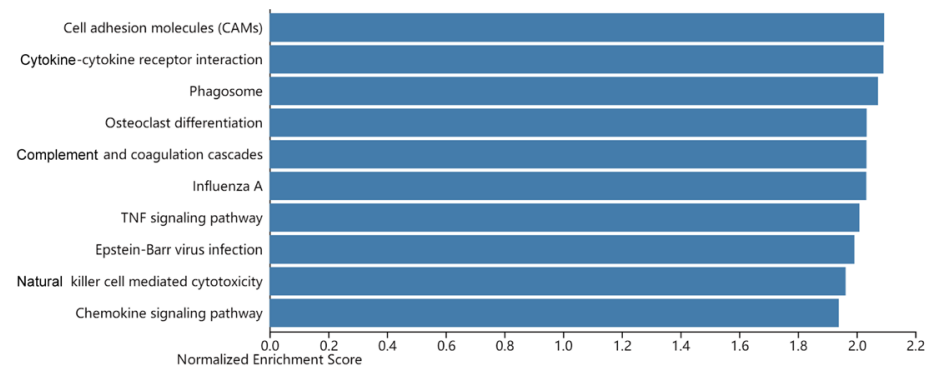

Fig. 2 Potential mechanisms of IL1RN in PTC. a Genes highly correlated with IL1RN identified by Pearson correlation analysis in the THCA cohort. b Heatmaps showing the top 50 genes positively and (c) negatively correlated with IL1RN in the TCHA cohort. d PPI of the top 100 significantly correlated genes. e GO biological process terms and (f) KEGG pathways significantly enriched in genes coexpressed with IL1RN in the THCA cohort

(Fig. 5e), pancreatic adenocarcinoma (PADD) (Fig. 5f) and uterine corpus endometrial carcinoma (UCEC) (Fig. 5g).

\section{Discussion}

In this study, we first revealed higher IL1RN expression in PTC tissue compared than in normal tissues and its diagnostic and prognostic value by integrated bioinformatics analysis. To further explore the potential function of IL1RN, we identified genes related to IL1RN expression, and functional enrichment analyses were conducted. The results of the KEGG pathway and GO analyses revealed the significant enrichment of genes in immune-related pathways. Our work further demonstrated that high IL1RN expression was significantly associated with decreased 

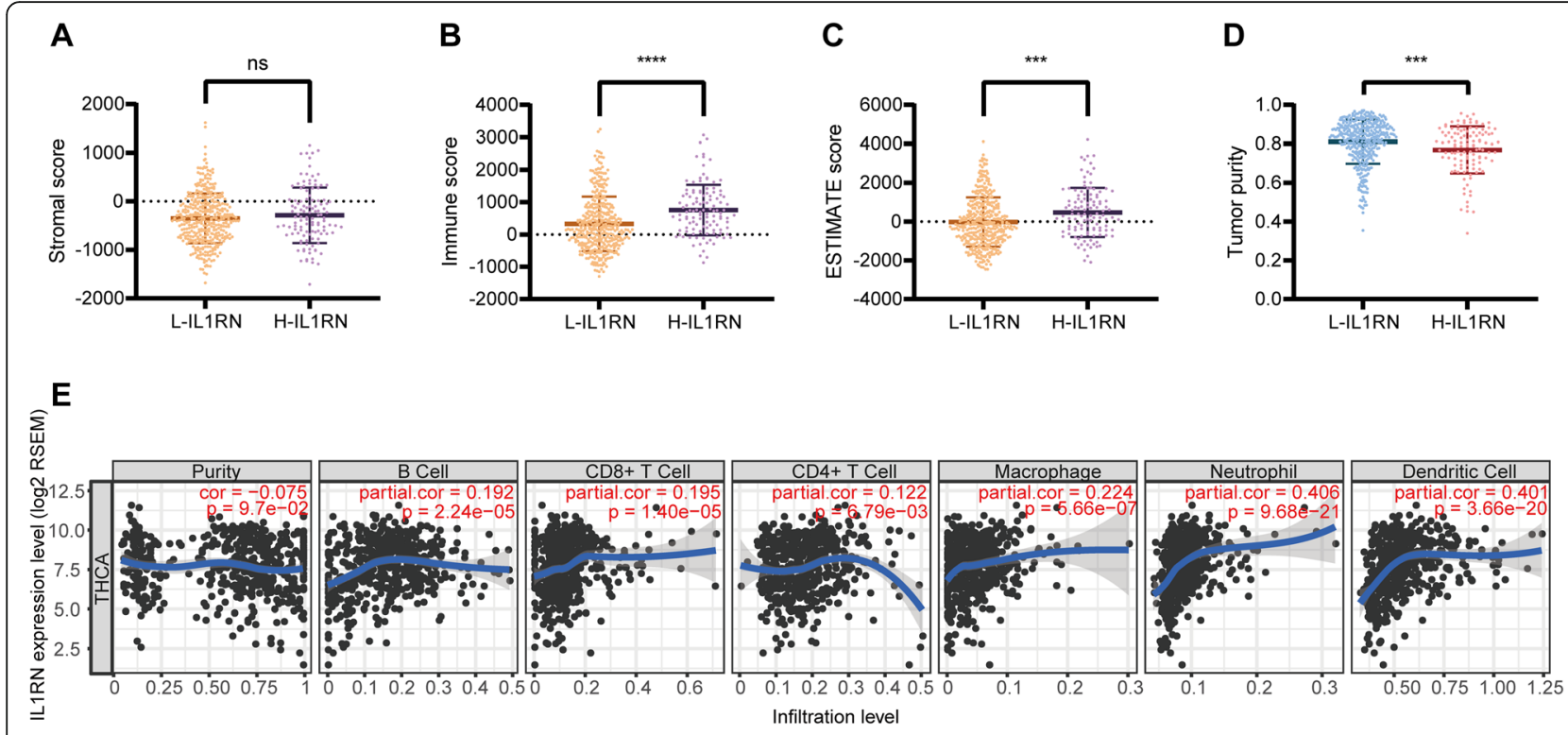

$\mathbf{F}$

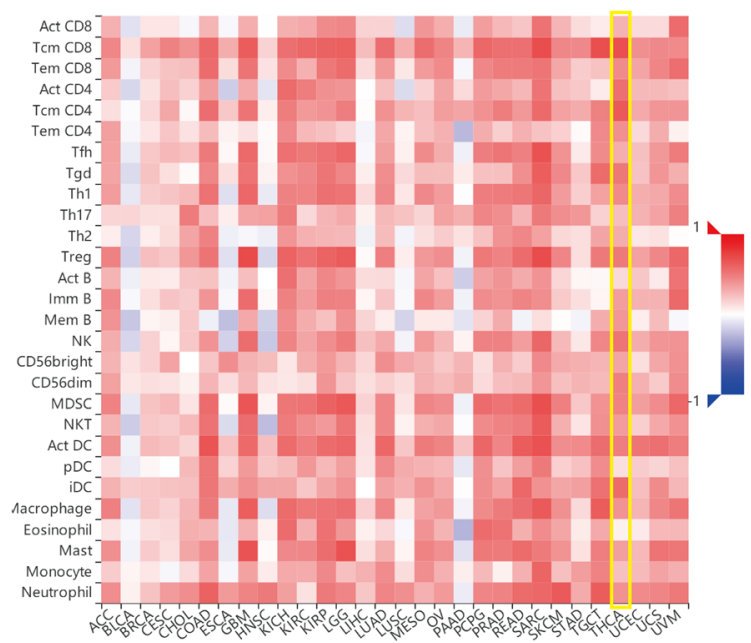

G

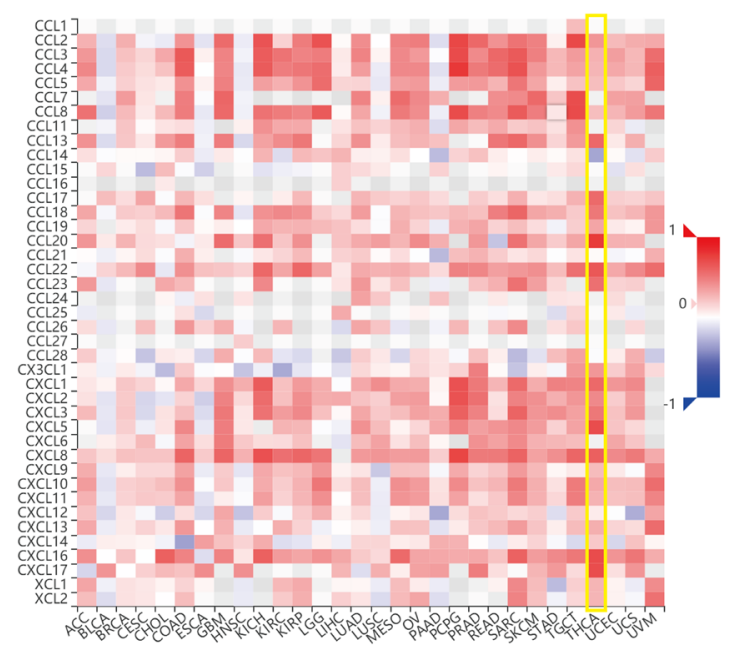

Fig. 3 ILIRN is closely related to immunity in PTC. ESTIMATE analysis of (a) stromal scores, (b) immune scores, (c) ESTIMATE scores, and (d) tumor purity between the L-IL1RN and H-IL1RN groups. e TIMER analysis of purity-corrected partial Spearman correlations between the expression of IL1RN and the infiltration of six types of immune cells in the THCA cohort. $\mathbf{f}$ Correlation analysis between the expression of IL1RN and 28 types of TILs across human cancers via TISIDB. $\mathbf{g}$ Correlation analysis between the expression of IL1RN and the levels of chemokines across human cancers via TISIDB

tumor purity and increased immune infiltration. Through methylation-related analysis, we determined that DNA methylation might be a regulatory mechanism of IL1RN. In addition, the analysis of the relationship between IL1RN expression and PTC mutations showed that high IL1RN expression was associated with mutated BRAF, wild-type NRAS and wild-type HRAS. Finally, IL1RN shows superior prognostic value for various cancers according to the pancancer analysis.
Niedzwiecki et al. [21] revealed that the serum levels of IL1RN were upregulated in patients with ATC and PTC, but no statistically significant difference was found in PTC. We found that the expression of IL1RN was increased in PTC tissues compared with that in normal tissues in 1 TCGA cohort and 4 GEO cohorts and suggested that IL1RN might be used as a potential diagnostic biomarker in PTC. We guess that overexpression of IL1RN in thyroid tissues of PTC patients result in elevated levels of serum IL1RN. Thus, the 
A

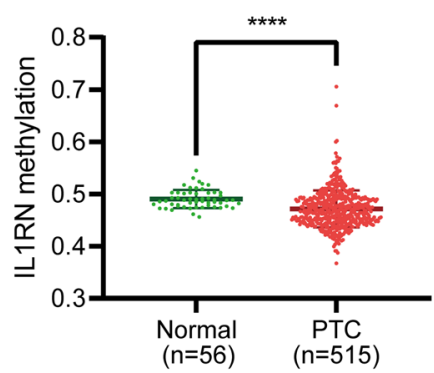

B

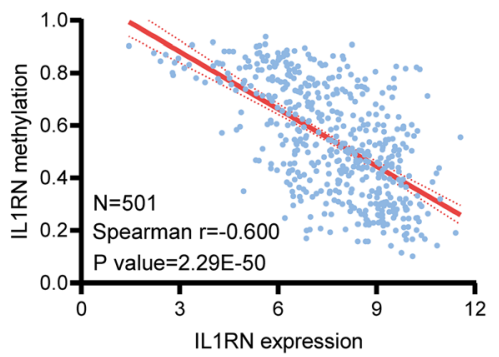

C

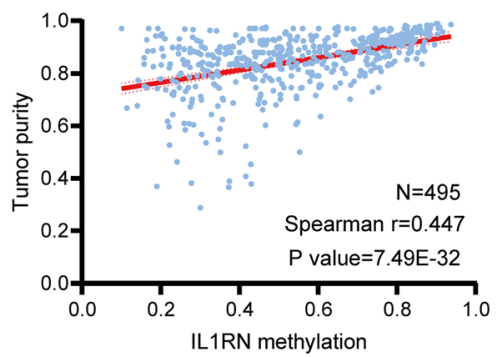

E

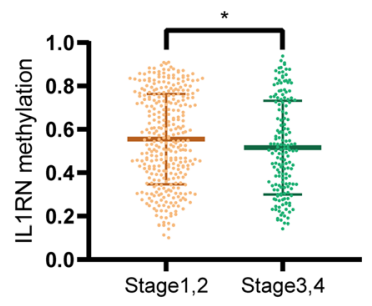

$\mathbf{F}$

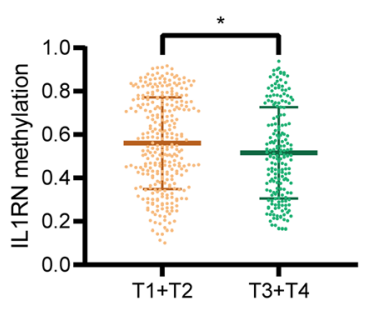

G

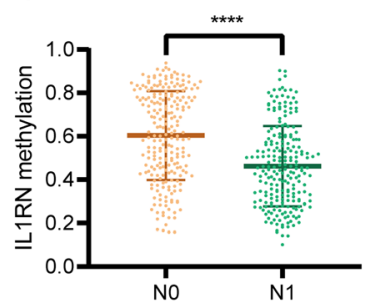

H

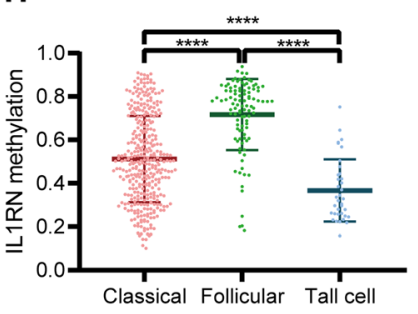

I

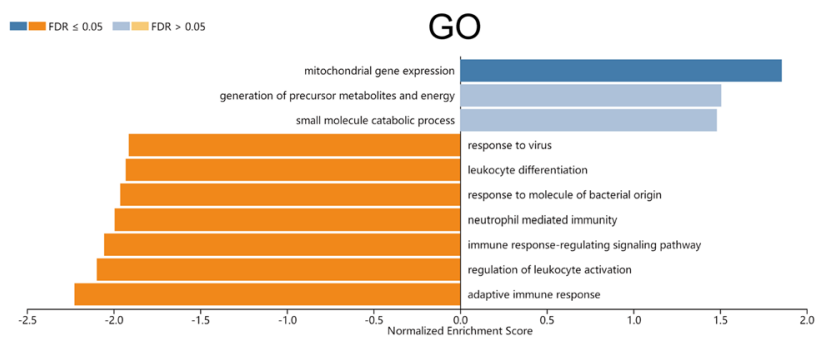

G

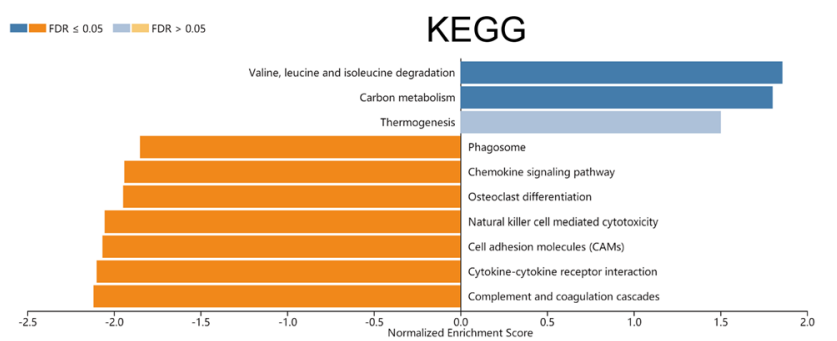

Fig. 4 Methylation of IL1RN in PTC. a IL1RN methylation levels were compared between PTC and normal tissues. $\mathbf{b}$ Correlation analysis between IL1RN methylation and IL1RN expression in PTC. c Correlation analysis between IL1RN methylation and tumor purity in PTC. IL1RN methylation level among patients with different (d) clinical stages, (e) T classifications, (f) N classifications, and (g) histological types of PTC. $\mathbf{h}$ GO biological process terms and (I) KEGG pathways significantly altered by IL1RN methylation in the THCA cohort

findings of Niedzwiecki et al. corroborate our results. Our results were consistent with those of previous reports on the upregulation of IL1RN in cervical carcinoma [13], gastric cancer [14], lung cancer [15], and endometrial cancer tissues [16]. These findings are also basically consistent with the results of our pancancer study. In contrast to these findings, IL1RN exhibited low expression in oral squamous cell carcinomas (OSCCs) [19].

The results of this study showed the value of IL1RN as a clinical biomarker in PTC and emphasized its potential as a prognostic biomarker in PTC patients. PTC patients with high IL1RN expression had decreased PFS compared to those with low IL1RN expression. Furthermore, high expression of IL1RN was significantly correlated with clinical stage, lymph node metastasis and pathological type. Various studies have highlighted the significant association between IL1RN expression and poor cancer prognosis; however, the results of these studies are conflicting. Some studies revealed that there was a significant positive correlation between poor prognosis and the expression of IL1RN [14, 16, 28]. In contrast, low levels of IL1RN have been associated with increased disease severity in myeloma [29], colorectal cancer [30] and prostate cancer [31]. 
Table 3 Spearman correlation between IL1RN methylation sites and IL1RN expression

\begin{tabular}{lll}
\hline Methylation site & Spearman $\boldsymbol{r}$ & $\boldsymbol{P}$ value \\
\hline cg01467417 & -0.597 & $7.76 \mathrm{E}-50$ \\
cg02543462 & -0.406 & $2.53 \mathrm{E}-21$ \\
cg01991967 & -0.19 & $1.81 \mathrm{E}-05$ \\
cg03989987 & -0.164 & $2.28 \mathrm{E}-04$ \\
$\operatorname{cg} 10938446$ & -0.116 & 0.009 \\
$\operatorname{cg} 03703171$ & -0.107 & 0.016 \\
$\operatorname{cg} 11783497$ & -0.1 & 0.016 \\
$\operatorname{cg} 17669033$ & 0.027 & 0.548 \\
$\operatorname{cg} 23041410$ & -0.022 & 0.625 \\
$\operatorname{cg} 06658391$ & 0.018 & 0.687 \\
$\operatorname{cg} 25928199$ & NA & NA \\
cg25265126 & NA & NA \\
cg02377053 & NA & NA \\
\hline
\end{tabular}

The underlying mechanism of IL1RN in cancer development and progression is complicated and unclear. In this study, functional enrichment analysis of genes coexpressed with IL1RN showed that IL1RN participates in immune-related biological processes. Consistent with our findings, previous studies have focused on the role of IL1RN in tumor immunity. A study of gastric carcinoma [14] pointed out that on the one hand, IL1RN may promote tumor growth via the impairment of cellular immunity; on the other hand, IL1RN enables malignant cells to escape host immune responses. Smith, D. R. et al. [15] revealed that increased IL1RN in bronchogenic carcinoma is not accompanied by increased IL-1 $\beta$ activity. The altered balance of IL1RN and IL$1 \beta$ may result in impaired immune surveillance and cytotoxic activity. An experimental study of human glioblastoma cells showed that IL1RN secreted by tumor cells can counteract IL-1 function, which represents a potential escape mechanism that supports cancer growth [32]. In our study, IL1RN was significantly positively correlated with lymph node metastasis and tumor stage, so we speculated that IL1RN might also promote tumor aggressiveness and poor prognosis through immune-related mechanisms in PTC.

IL1RN is an endogenous natural antagonist of IL-1 [8], so the discovery of the interaction between IL1RN and IL-1 family molecules is a breakthrough in exploring the function of IL1RN. Onozaki et al. [33] reported that IL-1 is a cytocidal factor against several tumor cell lines. IL-1 may enhance cytotoxic $\mathrm{T}$ cell activity [34], the tumoricidal capacities of natural killer (NK) cells [35], and monocyte-mediated cytotoxicity [36]. Because IL-1 is critical for tumor immunity, elevation of IL1RN expression may result in a general environment favourable to tumor cells and enhance the metastatic and recurrence potential of tumors by changing local IL-1-dependent pathways.

IL-1 $\beta$ has been reported as an anticancer factor that acts to suppress proliferation and reduce the invasive potential of human PTC cells [37]. sIL-1ra has been shown to block IL-1 function by binding to IL-1 receptors at the cell membrane level [34]. IcIL-1ra is postulated to inhibit intracellular IL-1 activity [38]. Therefore, whether IL1RN can block the function of IL-1 and inhibit the anticancer effect of IL-1 $\beta$ in PTC is worth further study.

In our study, IL1RN showed a significant positive correlation with immune cells, which may be because a variety of immune cells can produce IL1RN through the stimulation of cytokines. Neutrophils produce IL1RN in response to granulocyte/macrophage colony stimulating factor (GM-CSF) and tumor necrosis factor- $\alpha$ (TNF- $\alpha$ ) [39]. IL-4 and IL-10 have been reported to increase the production of IL1RN by human monocytes [36]. Yanagawa, H.et, al. reported that IL-13 increases IL1RN production by human alveolar macrophages [40]. It was reported that the apoptotic cell death of monocytes was enhanced by administration of recombinant IL1RN [41]. Our study also showed a significant correlation between IL1RN expression and various cytokines, which implied that IL1RN may play a complex role in tumor immunity.

Our study suggests that methylation is a pretranscriptional regulatory mechanism for IL1RN. The methylation level of IL1RN was negatively correlated with its expression level and was also correlated with the disease stage, lymph node metastasis, and pathological type.

IL1RN is likely to be a potential biomarker associated with the diagnosis and prognosis of PTC. However, although the diagnostic value, prognostic value and molecular functions of IL1RN in PTC have been analyzed through bioinformatics methods, the conclusions have not yet been confirmed by experiments. Therefore, further research is necessary to explore the role of IL1RN in PTC and the pharmacological value of IL1RN as a therapeutic target.

\section{Conclusions}

IL1RN is a good prognostic and diagnostic biomarker of PTC. IL1RN may promote thyroid cancer progression through immune-related pathways. Methylation may act as an upstream regulator of IL1RN 
A

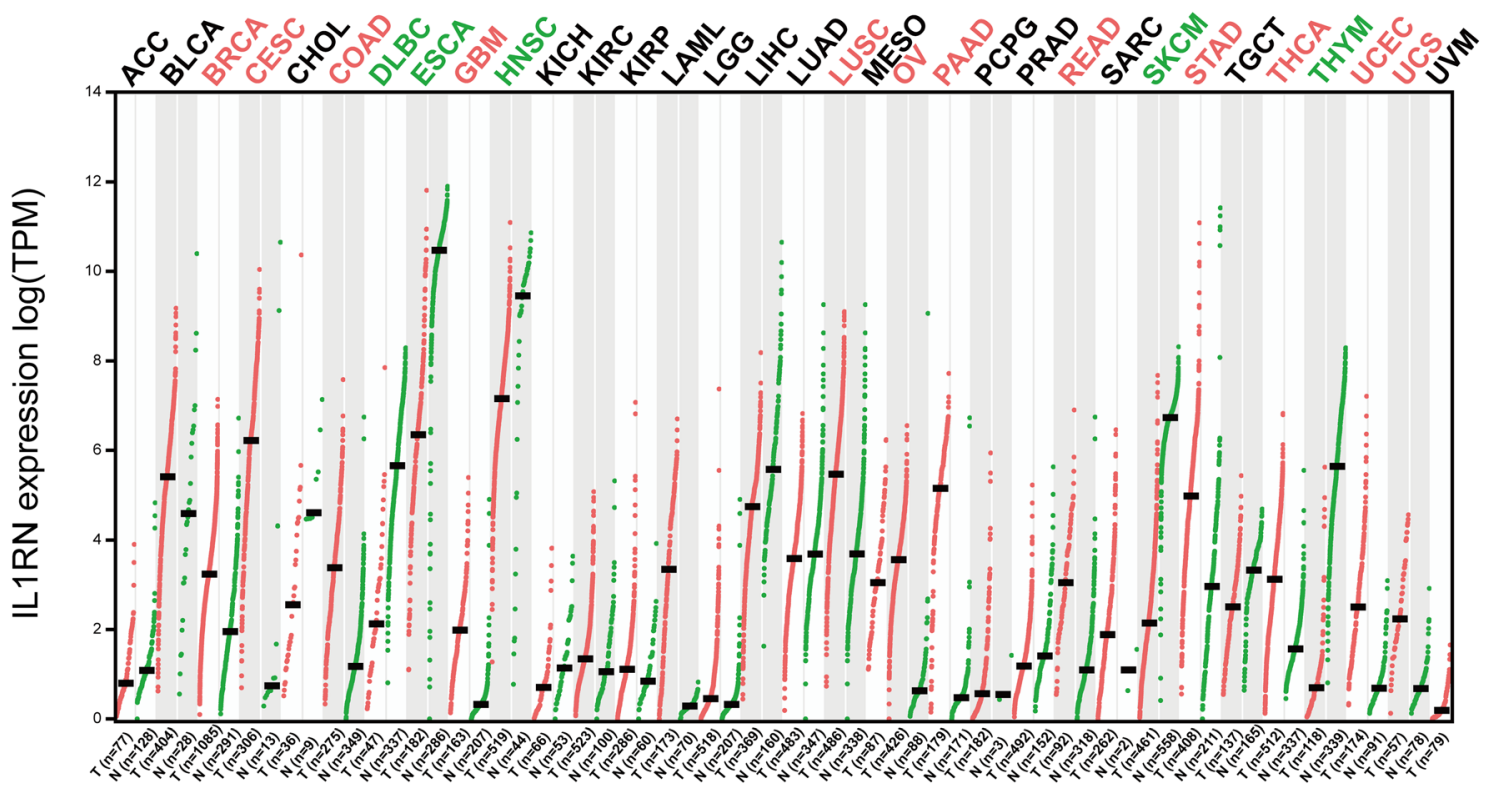

B

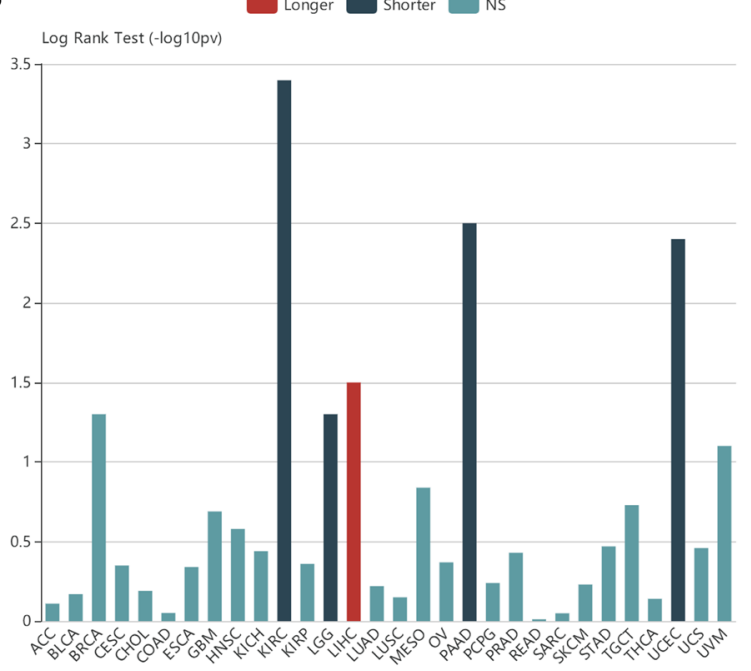

C

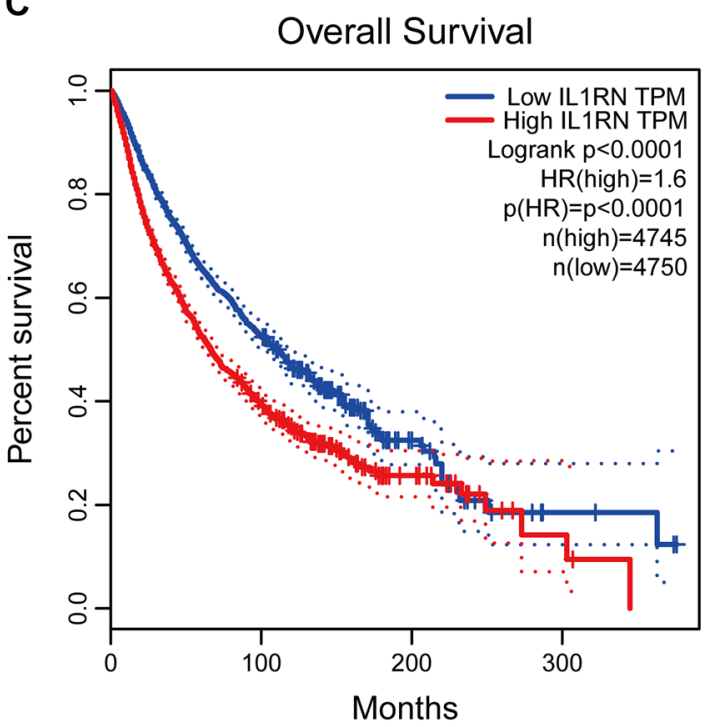

F

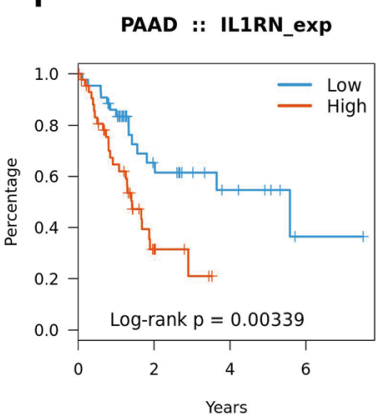

G

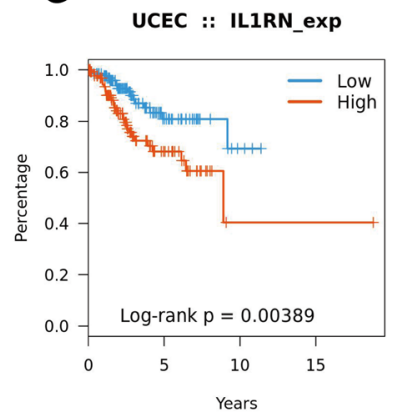

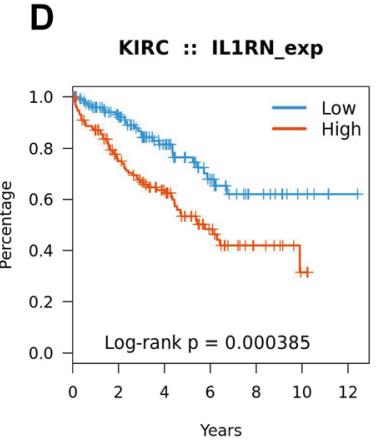

E

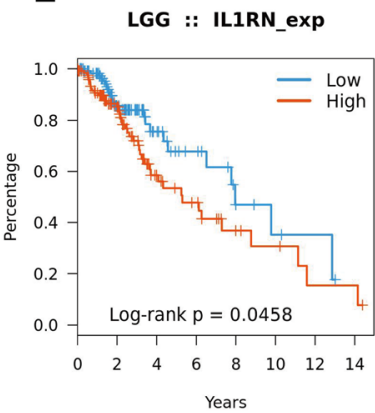

Fig. 5 Generalization value of IL1RN across cancer. a Comparison of IL1RN mRNA expression between cancer and paracancerous tissuess across cancers. b Associations between IL1RN expression and OS across human cancers. K-M survival analysis between the L-IL1RN and H-IL1RN in (c) pan-cancer cohorts, d KIRC, e LGG, f PAAD, and $\mathbf{g} U C E C$ 
expression and biological function. Additionally, IL1RN showed broad prognostic value in an analysis of a pancancer cohort.

\section{Supplementary information}

The online version contains supplementary material available at https://doi. org/10.1186/s12885-020-07620-8.

Additional file 1: Fig. S1. IL1RN expression is closely related to immunity across human cancers. Correlation analysis between the expression of ILIRN and the levels of (A) immune activating cytokines, (B) immune suppressive cytokines, (C) MHCs, and (D) chemokine receptors.

Additional file 2: Fig. S2. Relationship between ILIRN expression and gene mutations in PTC. (A) Volcano plot of IL1RN expression and gene mutations in THCA. Relationship between IL1RN expression and (B) BRAF mutation, (C) NRAS mutation, and (D) HRAS mutation.

\begin{abstract}
Abbreviations
IL1RN: Interleukin-1 receptor antagonist; PTC: Papillary thyroid carcinoma; TCGA: The Cancer Genome Atlas; OS: Overall survival; GEPIA: Gene Expression Profiling Interactive Analysis; PFS: Progression-free survival; THCA: Thyroid carcinoma; IL-1: Interleukin-1; sIL-1ra: The soluble extracellular form of IL1RN; iclL-1ra: The intracellular form of IL1RN; ATC: Anaplastic carcinoma; FTC: Follicular carcinoma; NCBI: The National Center for Biotechnology Information; GEO: Gene Expression Omnibus; H-IL1RN: High IL1RN expression group; L-IL1RN: Low IL1RN expression group; PPI: Protein-protein interaction; sSGSEA: Single-sample gene-set enrichment analysis; TIMER: Tumor Immune Estimation Resource; DCs: Dendritic cells; MHC: Major histocompatibility complex; K-M: Kaplan-Meier; CAMs: Cell adhesion molecules; KIRC: Kidney renal clear cell carcinoma; LGG: Lower grade glioma; PADD: Pancreatic adenocarcinoma; UCEC: Uterine corpus endometrial carcinoma; OSCCs: Oral squamous cell carcinomas; NK: Natural killer; GM-CSF: Granulocyte/ macrophage colony stimulating factor; TNF-a: Tumor necrosis factor-a
\end{abstract}

\section{Acknowledgements}

We would like to acknowledge the public databases including TCGA and GEO for their contributions to human medicine.

\section{Authors' contributions}

ZX designed the analytical strategies, performed data analyses and wrote the manuscript. XL performed data analysis and wrote the manuscript. $Y Z H, S W$, SYW, JS, YCH, YL and SJX performed data analysis. JZ conceived the research and wrote the manuscript. The authors have read and approved the manuscript.

\section{Funding}

This research was supported by National Natural Science Foundation of China (81600602). The funding bodies played no role in the design of the study and collection, analysis, and interpretation of data and in writing the manuscript.

\section{Availability of data and materials}

The datasets analysed during the current study are available in The Cancer Genome Atlas (TCGA) (https://tcga-data.nci.nih.gov/tcga/) or the National Center for Biotechnology Information (NCBI) Gene Expression Omnibus (GEO) database (http://www.ncbi.nlm.nih.gov/geo/). The raw data may be made available upon reasonable request from the corresponding authors.

\section{Ethics approval and consent to participate}

Not applicable.

\section{Consent for publication}

Not applicable.

\section{Competing interests}

The authors declare that they have no competing interests.
Received: 3 July 2020 Accepted: 8 November 2020

Published online: 25 November 2020

\section{References}

1. Bray F, Ferlay J, Soerjomataram I, Siegel RL, Torre LA, Jemal A. Global cancer statistics 2018: GLOBOCAN estimates of incidence and mortality worldwide for 36 cancers in 185 countries. CA Cancer J Clin. 2018:68(6):394-424

2. Seib CD, Sosa JA. Evolving understanding of the epidemiology of thyroid cancer. Endocrinol Metab Clin North Am. 2019;48(1):23-35.

3. Siegel R, Naishadham D, Jemal A. Cancer statistics, 2013. CA Cancer J Clin. 2013;63(1):11-30.

4. Cancer Genome Atlas Research N. Integrated genomic characterization of papillary thyroid carcinoma. Cell. 2014;159(3):676-90.

5. Haugen BR, Alexander EK, Bible KC, Doherty GM, Mandel SJ, Nikiforov YE, et al. 2015 American Thyroid Association management guidelines for adult patients with thyroid nodules and differentiated thyroid cancer: the American Thyroid Association guidelines task force on thyroid nodules and differentiated thyroid cancer. Thyroid. 2016;26(1):1-133.

6. Raue F, Frank-Raue K. Thyroid cancer: risk-stratified management and individualized therapy. Clin Cancer Res. 2016;22(20):5012-21.

7. Sims JE, Smith DE. The IL-1 family: regulators of immunity. Nat Rev Immunol. 2010;10(2):89-102.

8. Arend WP. Interleukin 1 receptor antagonist. A new member of the interleukin 1 family. J Clin Invest. 1991;88(5):1445-51.

9. Arend WP, Malyak M, Guthridge CJ, Gabay C. Interleukin-1 receptor antagonist: role in biology. Annu Rev Immunol. 1998;16:27-55.

10. Arend WP. The balance between IL-1 and IL-1Ra in disease. Cytokine Growth Factor Rev. 2002;13(4-5):323-40.

11. Ricote M, Garcia-Tunon I, Bethencourt FR, Fraile B, Paniagua R, Royuela M. Interleukin-1 (IL-1alpha and IL-1beta) and its receptors (IL-1RI, IL-1RII, and IL$1 \mathrm{Ra}$ ) in prostate carcinoma. Cancer. 2004;100(7):1388-96.

12. Hsieh TC, Chiao JW. Growth modulation of human prostatic cancer cells by interleukin-1 and interleukin-1 receptor antagonist. Cancer Lett. 1995;95(12):119-23.

13. Fujiwaki R, lida K, Nakayama K, Kanasaki H, Hata K, Katabuchi H, et al. Clinical significance of interleukin-1 receptor antagonist in patients with cervical carcinoma. Gynecol Oncol. 2003:89(1):77-83.

14. lizuka N, Hazama S, Hirose K, Abe T, Tokuda N, Fukumoto T, et al. Interleukin-1 receptor antagonist mRNA expression and the progression of gastric carcinoma. Cancer Lett. 1999;142(2):179-84.

15. Smith DR, Kunkel SL, Standiford TJ, Chensue SW, Rolfe MW, Orringer MB, et al. The production of interleukin-1 receptor antagonist by human bronchogenic carcinoma. Am J Pathol. 1993;143(3):794-803.

16. Van Le L, Haskill S, Jaffe GJ, Fowler WC Jr. Expression of interleukin-1 and interleukin-1 receptor antagonists in endometrial cancer. Gynecol Oncol. 1991;42(2):161-4.

17. Yanagawa $\mathrm{H}$, Yano S, Haku T, Ohmoto $Y$, Sone S. Interleukin-1 receptor antagonist in pleural effusion due to inflammatory and malignant lung disease. Eur Respir J. 1996;9(6):1211-6.

18. Mustea A, Pirvulescu C, Konsgen D, Braicu El, Yuan S, Sun P, et al. Decreased IL1 RA concentration in ascites is associated with a significant improvement in overall survival in ovarian cancer. Cytokine. 2008;42(1):77-84.

19. Shiiba M, Saito K, Yamagami H, Nakashima D, Higo M, Kasamatsu A, et al. Interleukin-1 receptor antagonist (IL1RN) is associated with suppression of early carcinogenic events in human oral malignancies. Int J Oncol. 2015; 46(5):1978-84.

20. Wetzler M, Kurzrock R, Estrov Z, Kantarjian $H$, Gisslinger $H$, Underbrink MP, et al. Altered levels of interleukin-1 beta and interleukin-1 receptor antagonist in chronic myelogenous leukemia: clinical and prognostic correlates. Blood. 1994;84(9):3142-7.

21. Niedzwiecki S, Stepien T, Kuzdak K, Stepien H, Krupinski R, Seehofer D, et al. Serum levels of interleukin-1 receptor antagonist (IL-1 ra) in thyroid cancer patients. Langenbecks Arch Surg. 2008;393(3):275-80.

22. Budczies J, Klauschen F, Sinn BV, Gyorffy B, Schmitt WD, Darb-Esfahani $S$, et al. Cutoff finder: a comprehensive and straightforward web application enabling rapid biomarker cutoff optimization. PLoS One. 2012;7(12):e51862.

23. Vasaikar SV, Straub P, Wang J, Zhang B. LinkedOmics: analyzing multi-omics data within and across 32 cancer types. Nucleic Acids Res. 2018;46(D1): D956-63. 
24. Yoshihara K, Shahmoradgoli M, Martinez E, Vegesna R, Kim H, Torres-Garcia $W$, et al. Inferring tumour purity and stromal and immune cell admixture from expression data. Nat Commun. 2013;4:2612.

25. Li T, Fan J, Wang B, Traugh N, Chen Q, Liu JS, et al. TIMER: a web server for comprehensive analysis of tumor-infiltrating immune cells. Cancer Res. 2017; 77(21):e108-10.

26. Ru B, Wong CN, Tong Y, Zhong JY, Zhong SSW, Wu WC, et al. TISIDB: an integrated repository portal for tumor-immune system interactions. Bioinformatics. 2019:35(20):4200-2.

27. Tang Z, Li C, Kang B, Gao G, Li C, Zhang Z. GEPIA: a web server for cancer and normal gene expression profiling and interactive analyses. Nucleic Acids Res. 2017;45(W1):W98-W102.

28. Ito H, Miki C. Profile of circulating levels of interleukin-1 receptor antagonist and interleukin-6 in colorectal cancer patients. Scand J Gastroenterol. 1999; 34(11):1139-43.

29. Gherardi RK, Belec L, Soubrier M, Malapert D, Zuber M, Viard JP, et al. Overproduction of proinflammatory cytokines imbalanced by their antagonists in POEMS syndrome. Blood. 1996;87(4):1458-65.

30. Iwagaki H, Hizuta A, Tanaka N. Interleukin-1 receptor antagonists and other markers in colorectal cancer patients. Scand J Gastroenterol. 1997:32(6):577-81.

31. Parekh DJ, Ankerst DP, Baillargeon J, Higgins B, Platz EA, Troyer D, et al. Assessment of 54 biomarkers for biopsy-detectable prostate cancer. Cancer Epidemiol Biomarkers Prev. 2007;16(10):1966-72.

32. Oelmann E, Kraemer A, Serve H, Reufi B, Oberberg D, Patt S, et al. Autocrine interleukin-1 receptor antagonist can support malignant growth of glioblastoma by blocking growth-inhibiting autocrine loop of interleukin-1. Int J Cancer. 1997;71(6):1066-76.

33. Onozaki K, Matsushima K, Aggarwal BB, Oppenheim JJ. Human interleukin 1 is a cytocidal factor for several tumor cell lines. J Immunol. 1985;135(6): 3962-8.

34. Hannum CH, Wilcox CJ, Arend WP, Joslin FG, Dripps DJ, Heimdal PL, et al. Interleukin-1 receptor antagonist activity of a human interleukin-1 inhibitor. Nature. 1990;343(6256):336-40.

35. Voronov E, Weinstein Y, Benharroch D, Cagnano E, Ofir R, Dobkin M, et al. Antitumor and immunotherapeutic effects of activated invasive $T$ lymphoma cells that display short-term interleukin 1alpha expression. Cancer Res. 1999;59(5):1029-35.

36. de Waal Malefyt R, Abrams J, Bennett B, Figdor CG, de Vries JE. Interleukin 10(IL-10) inhibits cytokine synthesis by human monocytes: an autoregulatory role of IL-10 produced by monocytes. J Exp Med. 1991; 174(5):1209-20.

37. Yip I, Pang XP, Berg L, Hershman JM. Antitumor actions of interferongamma and interleukin-1 beta on human papillary thyroid carcinoma cell lines. J Clin Endocrinol Metab. 1995;80(5):1664-9.

38. Mosley B, Urdal DL, Prickett KS, Larsen A, Cosman D, Conlon PJ, et al. The interleukin-1 receptor binds the human interleukin-1 alpha precursor but not the interleukin-1 beta precursor. J Biol Chem. 1987;262(7):2941-4.

39. McColl SR, Paquin R, Menard C, Beaulieu AD. Human neutrophils produce high levels of the interleukin 1 receptor antagonist in response to granulocyte/macrophage colony-stimulating factor and tumor necrosis factor alpha. J Exp Med. 1992;176(2):593-8.

40. Lovett D, Kozan B, Hadam M, Resch K, Gemsa D. Macrophage cytotoxicity: interleukin 1 as a mediator of tumor cytostasis. J Immunol. 1986;136(1):340-7.

41. Furukawa Y, Kikuchi J, Terui Y, Kitagawa S, Ohta M, Miura Y, et al. Preferential production of interleukin-1 beta over interleukin-1 receptor antagonist contributes to proliferation and suppression of apoptosis in leukemic cells. Jpn J Cancer Res. 1995;86(2):208-16.

\section{Publisher's Note}

Springer Nature remains neutral with regard to jurisdictional claims in published maps and institutional affiliations.

Ready to submit your research? Choose BMC and benefit from:

- fast, convenient online submission

- thorough peer review by experienced researchers in your field

- rapid publication on acceptance

- support for research data, including large and complex data types

- gold Open Access which fosters wider collaboration and increased citations

- maximum visibility for your research: over $100 \mathrm{M}$ website views per year

At BMC, research is always in progress.

Learn more biomedcentral.com/submissions 